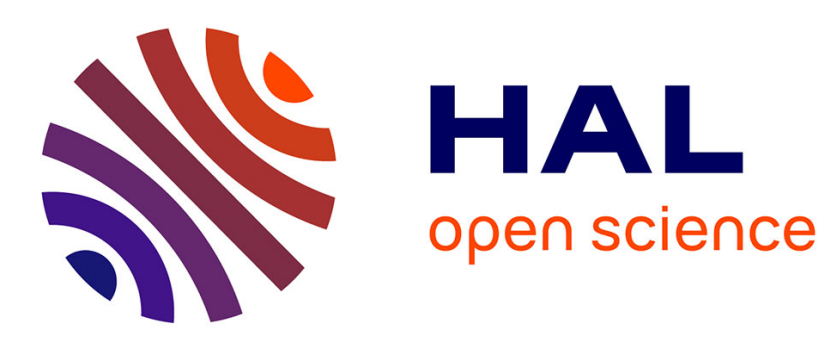

\title{
Les bibliothèques universitaires se désabonnent
}

\author{
Claire Nguyen
}

\section{To cite this version:}

Claire Nguyen. Les bibliothèques universitaires se désabonnent. Documentaliste - Sciences de l'Information, 2014, 51 (3), pp.9-11. sic_01070117v2

\section{HAL Id: sic_01070117 \\ https://archivesic.ccsd.cnrs.fr/sic_01070117v2}

Submitted on 8 Oct 2014

HAL is a multi-disciplinary open access archive for the deposit and dissemination of scientific research documents, whether they are published or not. The documents may come from teaching and research institutions in France or abroad, or from public or private research centers.
L'archive ouverte pluridisciplinaire HAL, est destinée au dépôt et à la diffusion de documents scientifiques de niveau recherche, publiés ou non, émanant des établissements d'enseignement et de recherche français ou étrangers, des laboratoires publics ou privés. 
est maintenant possible ${ }^{8}$. Ces systèmes sont utilisés pour s'entraîner à la prise de décision dans des environnements socio- techniques complexes et difficiles d'accès (analyse de risque, gestion de crise, etc.). Le projet Humans (HUman Models based Artificial eNvironments Software platform) vise à modéliser l'activité humaine et proposer différents scénarios (situations d'apprentissage) intégrant le décor et les personnages capables d'exprimer des émotions. II utilise des techniques de traitement d'image associé aux systèmes multi-agents (intelligence artificielle).

\section{Élargir les points de vue}

Pour conclure cette conférence, une table ronde, animée par JeanPaul Langlois, président d'IMdR, a abordé des thèmes relatifs aux stratégies pour réduire les risques de perte de connaissances, les principales difficultés de mise en œuvre, les conditions de réussite d'une démarche KM et la motivation des dirigeants et des collaborateurs.

Pour Grégoire Postel-Vinay, du ministère du Redressement productif, les connaissances sous des formes différentes constituent un levier pour sortir de la crise par l'innovation. II s'agit également de savoir exploiter les Big data. On retiendra son analogie aux deux représentations de la déesse Athéna, tantôt comme une chouette (allégorie des connaissances et de la sagesse), tantôt comme une guerrière casquée, prête à protéger le savoir et les connaissances.

André Lannoy

Vice-président IMdR à la Stratégie et aux activités andre.lannoy@orange.fr

$>$ Eunika Mercier-Laurent Directeur stratégique Global Innovation Strategies eunika@innovation3d.fr

$>$ Bertrand de Miramon IMdR

$>$ John Mitchel Obama Délégué technique IMdR john.obama@imdr.eu

\section{Les bibliothèques universitaires se désabonnent}

\author{
[ publications ] Victimes de la crise, les bibliothèques \\ universitaires revoient considérablement à la baisse le \\ nombre de leurs acquisitions. À partir d'éléments tirés de \\ rapports, d'articles et d'entretiens, l'article décrit les raisons \\ d'une telle situation, la manière dont ces bibliothèques \\ gèrent la pénurie et propose des perspectives.
}

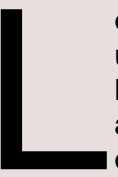

es coupes budgétaires importantes vécues par plusieurs universités ont conduit à des désabonnements spectaculaires aux revues scientifiques ou à une forte diminution des achats de monographies. La Bibliothèque interuniversitaire de Santé (BIU Santé), rattachée à l'université Paris Descartes, a dû suspendre la majorité de ses abonnements imprimés ou électroniques (revues, bases de données, livres électroniques) tout comme le Service commun de la documentation de Paris Descartes (SCD). Ce cas est symptomatique d'une situation générale dégradée provoquée par plusieurs facteurs.

\section{Les causes}

\section{La situation financière délicate des universités}

L'autonomie des universités ${ }^{1}$ donne aux établissements des responsabilités et compétences élargies en matière budgétaire et de gestion des ressources humaines. Les conséquences de cette autonomie n'ont pas toujours été bien mesurées. Malgré une dotation stable aux établissements, les transferts de charge entre l'État et l'université concernant la masse salariale n'ont pas été correctement anticipés. Nombre d'établissements gèlent les postes d'enseignants et des personnels administratifs, techniques et de bibliothèques ; dix-sept présidents ont voté une motion sur la situation financière des universités lors de leur dernière conférence?

Dans ce contexte difficile, les arbitrages nécessaires placent les bibliothèques universitaires dans une situation d'autant plus délicate que leur dotation n'est plus attribuée directement par le ministère de l'Enseignement supérieur et de la Recherche. En décembre 2013, le rapport de l'Agence d'évaluation de la recherche et de l'enseignement supérieur (Aeres) sur l'université Paris Descartes annonçait qu'une " une attention particulière devait être portée par les universités de tutelle de ces deux structures [BIU Santé et SCD] sur leur capacité à maintenir l'offre actuelle dans une période de réduction des ressources " ${ }^{3}$. Les enquêtes de l'Association des directeurs et personnels de direction des bibliothèques universitaires et de la documentation (ADBU) et du consortium Couperin démontrent que si la crise ne frappe de manière spectaculaire que quelques bibliothèques universitaires, les budgets documentaires sont en baisse depuis $2012(-3,3 \%)$. La baisse des moyens affectant les bibliothèques était de $9 \%$ en 2012, de $5 \%$ en 2013 et en $2014^{4}$. Les bibliothèques doivent aussi procéder à des arbitrages, imposés parfois par leur tutelle. Ainsi, ces baisses moyennes masquent une chute des achats de livres pénalisant les étudiants $(-25 \%$ entre 2011 et 2014) et, entre 2002 et 2014, une « explosion des dépenses de la documentation électronique destinée aux laboratoires : 
Les conséquences se font ressentir rapidement quand les éditeurs coupent les accès électroniques

+458\%, sans commune mesure avec la diminution logique des budgets consacrés aux revues imprimées (-54 \%) $»^{5}$. Depuis juillet 2011, les bibliothèques de l'université Paris 13 sont « dans l'incapacité d'acquérir la moindre documentation destinée aux étudiants des niveaux L1 à M1 [...] car tous les crédits disponibles ont été engloutis par les abonnements aux bouquets électroniques, dont les $4 / 5^{e}$ au moins sont de niveau recherche ${ }^{6}$.

\section{Les modèles économiques de I'IST}

La charge financière des abonnements aux revues et ressources électroniques a pesé lourdement dans la balance ${ }^{7}$. Le prix des revues a connu une inflation de $273 \%$ entre 1986 et $2004^{\circ}$. Par ailleurs, les modèles économiques des "Big Deals » des grands éditeurs (Elsevier, etc.) du début des années 2000 ont donné un large accès aux catalogues électroniques de ces éditeurs mais ils ont aussi figé des chiffres d'affaires, basés sur les abonnements papier souscrits au moment du Big Deal, et dont le montant augmente d'année en année.

Les bibliothèques de référence ayant des abonnements importants, comme les Cadist ${ }^{9}$, ont dû gager une grande partie de leur budget pour pérenniser l'accès à ces catalogues $(2 / 3$ du budget documentaire en 2013 pour la BIU Santé) et assister à l'érosion inexorable de leurs collections, au détriment de plus petits éditeurs. Certains éditeurs profitent de leur situation de monopole pour imposer des changements de modèle basés sur les statistiques d'usage. Par ailleurs, la situation française n'est pas adaptée aux tarifications appliquées par des éditeurs anglo-saxons, dont le modèle est inspiré du campus unique à l'américaine. Pour protester contre une hausse tarifaire ne tenant pas compte de cette spécificité ${ }^{10}$, l'Université Pierre et Marie Curie n'a pas renouvelé en 2014 son abonnement à la prestigieuse revue Science. Le manque de transparence des conditions accordées aux différents établissements a mené - dans un contexte financier difficile - l'université de Montréal à supprimer la majorité de ses abonnements à l'éditeur Wiley, déplorant que son contrat fût bien moins favorable que celui accordé à l'université voisine McGill ${ }^{11}$.

\section{La gestion de la pénurie Les choix ou non-choix documentaires}

$\mathrm{Si}$ la direction des bibliothèques (DBIST) de l'Université de Versailles Saint-Quentin-en Yvelines (UVSQ) a pu maintenir sa documentation électronique (principalement destinée à la recherche), elle n'a pas reçu un euro sur les 530000 demandés pour acheter des monographies et le budget de fonctionnement requis n'a été satisfait qu'à hauteur de $35 \%{ }^{12}$. De novembre 2013 à avril 2014, tous les achats ont été bloqués; seuls les abonnements pluriannuels pour lesquels l'université s'était engagée ont pu être souscrits. Rappelons que la DBIST avait entrepris depuis plusieurs années un travail de rationalisation (dédoublonnage, substitution du papier par l'électronique) qui ne laissait guère de marge pour des économies supplémentaires. Au SCD de l'Université des Antilles et de la Guyane ${ }^{13}$ où $400000 €$ d'économies étaient annoncés en 2014 , ce sont les ressources électroniques les moins consultées qui ont fait l'objet de désabonnements (notamment Wiley, Muse) ; de même, les conventions liant le SCD et les CHU ont dû être révisées à la baisse. La BIU Santé et le SCD Paris Descartes ont subi une coupe de $25 \%$ de leur budget total en 2014. La BIU Santé ${ }^{14}$, qui ne parvenait jusque là qu'à maintenir ses acquisitions de monographies à un niveau correct, a dû s'attaquer à son cœur de collection. Déjà, entre 2007 et 2013 , elle avait désabonné près de 650 périodiques. La logique a été purement comptable. Le SCD de Paris Descartes a quant à lui été contraint de baisser tous les postes de dépense ${ }^{15}$.

\section{Restrictions et}

restructurations des services

Ces sacrifices n'ont pas suffi. La Bibliothèque Clermont université $(\mathrm{BCU})^{16}$, qui maintient son offre documentaire malgré les difficultés budgétaires de ClermontUniversité et de l'Université BlaisePascal, doit renoncer à cinq ETP ${ }^{17}$ de contractuels en septembre 2014. Cette circonstance a accéléré la restructuration de la carte du réseau documentaire local (fermeture de 5 bibliothèques) et pose clairement la question du changement de paradigme (passage de la conception de bibliothèque attachée à chaque faculté à celle de centres de ressources, points nodaux sur chaque campus proposant de la documentation et une offre de services).

Le problème des horaires d'ouverture s'est également posé à I'UVSQ, avec la fermeture prévue du site de Saint-Quentin-enYvelines 4 samedis de l'année universitaire, et la menace de fermeture de la bibliothèque de Boulogne. Des services ont été interrompus (prêt entre bibliothèques). La gestion de la communication envers les usagers a été délicate à mener. La BIU Santé a décidé d'expliquer ces problèmes avec transparence sur son blog et les réseaux sociaux ${ }^{18}$. L'Université de Montréal a communiqué, chiffres à la clef, sur son désabonnement au bouquet de Wiley et a proposé une gratuité du prêt entre bibliothèques à ses usagers. Mais la communication s'avère plus que périlleuse quand se mêlent l'obligation de réserve, la gestion d'une arrivée impromptue d'une équipe de télévision, etc. 


\section{Conséquences sur les usagers et les personnels}

Les conséquences se font ressentir rapidement quand les éditeurs coupent les accès électroniques ou quand les bibliothèques ferment ; moins rapidement, mais de manière tout aussi sensible, quand les collections imprimées ne sont plus à jour. Quentin Dége $z^{19}$ témoigne de l'absence de manuels récents en médecine, ou des codes en droit, primordiaux pour les étudiants. Au SCD de Nantes ${ }^{20}$, les livres manquent pour préparer le Capes et l'agrégation. À Paris Descartes, un outil destiné à la révision de l'internat a été supprimé, obligeant les étudiants à souscrire un abonnement individuel. Les collections financées par le dispositif Cadist sont compromises, tout comme la participation de la bibliothèque au plan régional de conservation partagé des périodiques médicaux. Les commentaires sur le blog de la BIU Santé sont autant de preuves de l'importance des bibliothèques dans la formation initiale et continue ${ }^{21}$. Les professionnels constatent les effets de la crise dans leur vie quotidienne : l'activité de traitement des acquisitions courantes en forte baisse, les conséquences sur le prêt entre bibliothèques à terme, des formations annulées.
Or, l'activité connaît aussi des pics en raison de la mise à jour des catalogues, de la communication envers usagers et éditeurs. Prenant leur mal en patience, les bibliothécaires de l'UVSQ mènent des chantiers au long cours (dons à traiter, catalogage des e-books). À la BIU Santé, les bibliothécaires ont sollicité des dons auprès des éditeurs qui ont quasiment tous accepté ; certains l'ont même proposé spontanément. Mais qu'en sera-t-il des années suivantes si le budget n'est pas augmenté ? Parallèlement, nombre d'usagers soutiennent leurs bibliothèques. À I'UVSQ, les étudiants ont livré une véritable campagne sur les réseaux sociaux ${ }^{22}$ pour soutenir leur bibliothèque, constituant ainsi sa meilleure défense.

\section{Quelles perspectives?}

L'amélioration budgétaire pérenne et structurelle est nécessaire mais non suffisante, car les modèles économiques actuels de I'IST ne sont plus soutenables. Couperin tente de les renégocier et de répartir les coûts équitablement entre les établissements dans le cadre de groupements de commande nationaux. Si l'on sort des modèles traditionnels, l'Open Access n'est pas forcément la panacée. Dans le modèle Gold, la charge des bibliothèques (abonnements) est transférée aux institutions publiantes (coûts de publication assumés par l'auteur). Des pays vont plus loin encore, en incitant la diffusion gratuite en libre accès (Green Open Access) des résultats des recherches financées sur fonds publics ${ }^{23}$. Le programme européen Horizon 2020 changerat-il la donne ? La France est en retard, malgré des initiatives prises en la matière : les portails HAL, les achats nationaux de ressources électroniques pérennes (Istex).

Pour le moment, les bibliothécaires assurent l'exercice délicat d'accompagner la transition vers ces nouveaux modèles tout en négociant les modèles en cours. Poursuivre le rapprochement des bibliothécaires avec les usagers permettra d'affronter au mieux la pénurie, de rationaliser la politique documentaire et de service, mais surtout de définir stratégiquement la place de I'IST dans les bibliothèques. Le changement passera aussi par une refonte du système d'évaluation des chercheurs, dont la voix pèse fortement dans la gouvernance des établissements.

\section{$>$ Claire Nguyen}

Responsable des périodiques et des ressources électroniques BIU Santé Négociatrice pour Couperin claire.nguyen@biusante.parisdescartes.fr
Autonomie engagée par la loi relative aux libertés et responsabilités des universités (LRU) en 2007.

2. www.cpu.fr/wp-content/uploads/2013/10/ Motion-relative-aux-moyens-desuniversit\%C3\%A9s-20131024-CPU.pdf

3. www.aeres-evaluation.fr/Etablissements/ UNIVERSITE-PARIS-DESCARTES

4. Lettre de cadrage qui rappelle que le projet de loi de finances 2014 annonce une augmentation consacrée aux « programmes immobiliers, aux bourses d'étudiants, et à la création de 1000 emplois d'enseignants supplémentaires. » www. couperin.org/relations-editeurs/lettre-de-cadrage 5. http://adbu.fr/wp-content/uploads/2014/03/ Enquête_ADBU_2014.pdf

6. Message de Dominique Baudin, «Une politique documentaire sans budget? ", ADBUForum, 26 septembre 2012, http://bbf.enssib.fr/ consulter/bbf-2013-01-0024-004

Voir le récent article de Dominique Stora, "Recherche publique, profits privés », Nouve Observateur, 8 mai 2014 ou encore celui de Sandrine Cabut et David Larousserie, «À qui appartient le savoir ? ", Le Monde , 28/02/2013 www.lemonde.fr/sciences/article/2013/02/28/a qui-appartient-le-savoir_1840797_1650684.html

8. Enquête citée sur le site de Sparc, www. sparc.arl.org/resources/journals

9. www.enssib.fr/le-dictionnaire/cadist

10. L'éditeur voulait appliquer une hausse tarifaire conséquente, basée sur le nombre de sites géographiques. Or, comme nombre d'universités françaises, I'UPMC est disséminée sur de nombreux sites.

www.bib.umontreal.ca/

communiques/20140115-DB-annulation-periodiques-wiley.htm

12. Entretien avec Dorothée Pain-Camus, responsable des ressources électroniques, 23 mai 2014.

3. Entretien avec Sylvain Houdebert, directeur du SCD de I'UAG, 23 mai 2014.

4. www2.biusante.parisdescartes.fr/wordpress/ index.php/contraintes-budgetaires-desabonnements-2014

5. http://parisdescartes.libguides.com/desabonnements2014
16. Entretien avec Olivier Legendre, adjoint au directeur de la BCU, et avec Fabrice Boyer directeur de la BCU, 12 juin 2014

\section{Emploi temps plein}

18. www2.biusante.parisdescartes.fr/wordpress 19. Entretien avec Quentin Dégez, étudiant en médecine et président d'Interassos USQV, 26 juin 2014

20. Voir l'article de Nathalie Brafman, «À Nantes, l'université accablée par les pénuries et un plan de redressement », Le Monde, 13/01/2014. www.lemonde.fr/societe/article/2014/01/13/anantes-I-universite-accablee-par-les-penuries-etun-plan-de-redressement 4346802 3224.html

www2.biusante.parisdescartes.fr/wordpress/ index.php/contraintes-budgetaires-desabonnements-2014

22. Touche pas à ma BU, https://www.facebook com/interassos.uvsq

23. Olivier Legendre, «L'abus de l'open access est-il nuisible à la science ? ", DocumentalisteSciences de l'information, vol. 50, n4, 2013 